\title{
Enhanced suppression of localization in a continuous random-dimer model
}

\author{
Angel Sánchez $\dagger$ and Francisco Domínguez-Adameł \\ † Escuela Politécnica Superior, Universidad Carlos III, C. Butarque 15, E-28911 Leganés, \\ Madrid, Spain \\ \$ Departamento de Física de Materiales, Universidad Complutense, Ciudad Universitaria, \\ E-28040 Madrid, Spain
}

Received 25 October 1993, in final form 21 February 1994

\begin{abstract}
We consider a one-dimensional Kronig-Penney model with randomly placed dimer impurities. We show that this model has infinitely many resonances (zeroes of the reflection coefficient) giving rise to extended states, instead of the one allowed resonance arising in random tight-binding models with paired correlated on-site energies. We present exact transfer-matrix numerical calculations supporting, both realizationwise and on average, the conclusion that the model has a very large number of extended states, which can be relevant in several physical contexts.
\end{abstract}

Since the one-dimensional Kronig-Penney model was introduced [1], it has been applied to many fields of physics, such as band structure and electron dynamics in ordered solids, impurity levels, localization phenomena in disordered solids and liquids [2], microelectronic devices [3,4], properties of layered superconductors [5], electronic transport in spontaneously dimerized solids (Peierls transition) [6], and quark tunnelling in onedimensional nuclear models [7]. A most remarkable feature of wavefunctions in disordered Kronig-Penney models is their strong localization in well defined regions of the lattice [2]. Similar results arise in one-dimensional tight-binding Hamiltonians with random interactions (Anderson localization [8]). Localization of all eigenstates by disorder in one-dimensional systems has often been viewed as an exact statement [9].

Three years ago, Dunlap, Wu and Phillips [10-12] (see [13] for a review) studied a tightbinding model (the so-called random-dimer model, RDM) in which the on-site energy takes on one of two possible values, one of which was assigned at random to pairs of lattice sites. They showed that for a certain energy the reffection coefficient of a single defect vanished, and that this resonance was preserved when a finite concentration of defects were randomly placed in the chain. This gave rise to a set of delocalized states proportional to the square root of the number of sites. As a consequence, in such a system electronic transport can take place almost ballistically, which is the opposite to the above-mentioned general belief. Similar results have been also shown to hold true for dilute binary alloys by Flores [14]. The conclusion of Dunlap et al that a large number of states were not localized has been further confirmed by perturbative calculations by Bovier [15], although very recently a contrary viewpoint has been held by Gangopadhyay and Sen [16]. The RDM has been generalized recently [17] to include more complex arrangements of defects, without suppressing the existence of many extended states. 
In this paper, we concern ourselves with a more physical continuous model, which we call the continuous random-dimer model (henceafter, CRDM). It has to be noticed that this system is not the continuum version of the RDM of Dunlap et al, as studied in [17]. We build our model in the following way: we start from a usual Kronig-Penney model, given by a potential of the form

$$
V(x)=\sum_{n=1}^{N} \lambda_{n} \delta\left(x-x_{n}\right) .
$$

We choose $\lambda_{n}>0$ and we take the positions of the delta functions to be regularly spaced, i.e. $x_{n}=n$. We allow only two values for $\lambda_{n}, \lambda$ and $\lambda^{\prime}$, with the additional constraint that $\lambda^{\prime}$ appears only in pairs of neighbouring sites. In this fashion, we have defined a continuous version of the tight-binding random-dimer model. The corresponding Schrödinger equation is then

$$
\left[-\frac{\mathrm{d}^{2}}{\mathrm{~d} x^{2}}+\sum_{n=1}^{N} \lambda_{n} \delta(x-n)\right] \Psi(x)=E \Psi(x) .
$$

We believe that our model is more realistic than the RDM since no tight-binding approach is involved, and since the $\delta$-function potential is a good candidate to model more structured interactions [18]. We will see in the following that there exists a number of energies for which the reflection coefficient at a single dimer vanishes. Because interference effects are more complex in this continuous model than in tight-binding approaches, it is a non-trivial task to elucidate whether these resonances will survive when several dimers are located at random along the lattice.

We now use the techniques of dynamical systems theory, as first used by Bellissard $e t$ al [19] (see also [20]) to construct the Poincare map associated with (2). It is important to stress that, by doing this reduction to an equivalent tight-binding set of equations, we are not losing any information at all, and the calculations remain exact. The resulting equations are

$$
\Psi_{n+1}+\Psi_{n-1}=\left[2 \cos \sqrt{E}+\lambda_{n} \frac{\sin \sqrt{E}}{\sqrt{E}}\right] \Psi_{n}
$$

where $\Psi_{n} \equiv \Psi(x=n)$. Notice that the energy enters in the equations in a rather complicated fashion. To proceed, we have to take into account in the first place the condition for an electron to be able to move in the perfect $\left(\lambda^{\prime}=\lambda\right)$ lattice, namely

$$
\left|\cos \sqrt{E}+\frac{\lambda}{2} \frac{\sin \sqrt{E}}{\sqrt{E}}\right| \leqslant 1
$$

this constraint gives the allowed energy values once $\lambda$ is fixed. On the other hand, we follow Dunlap et al and study the problem of a single-pair defect on an otherwise perfect chain. In our case, a straightforward application of the results in [10] leads to the following condition for the vanishing of the reflection coefficient:

$$
\cos \sqrt{E}+\frac{\lambda^{\prime}}{2} \frac{\sin \sqrt{E}}{\sqrt{E}}=0 .
$$

It is a matter of simple algebra to transform the two equations (4) and (5) into these other, more useful equations:

$$
\begin{aligned}
& -\frac{2}{\lambda^{\prime}}=\frac{\tan \sqrt{E}}{\sqrt{E}} \\
& |\cos \sqrt{E}| \leqslant \frac{\lambda^{\prime}}{\left|\lambda-\lambda^{\prime}\right|} .
\end{aligned}
$$


Restricting ourselves to the range $\lambda \leqslant 2 \lambda^{\prime}$, equation $(6 b)$ is trivially verified, and therefore it poses no restrictions on the allowed energy values, aside from the fact that they must be positive. Hence, we are left only with $(6 a)$ to select the energy values for which the reflection coefficient of a single defect becomes exactly zero. As $\tan (\sqrt{E})$ is a $\pi$-periodic function and takes all values in $[-\infty,+\infty]$, for any $\lambda^{\prime}$ we choose we will find energy values solving for $\sqrt{E}$ in $(6 a)$ in every interval [ $(2 n-1) \pi / 2,(2 n+1) \pi / 2], n=1,2, \ldots$, i.e. we will have an infinite countable set of energies for which the single defect reflection coefficient vanishes. This is to be compared with the result of Dunlap et al, who found a unique energy in the allowed band (recall that their model is a single-band one) for which the same perfect transmission took place in the RDM. We discuss this point further in our conclusions.

We now proceed to the problem of the disordered lattice, containing a certain number of pair defects. To this end, we go back to (2) and introduce the reflection and transmission amplitudes through the relationships,

$$
\Psi(x)= \begin{cases}\mathrm{e}^{\mathrm{i} \sqrt{E} x}+R_{N} \mathrm{e}^{-\mathrm{i} \sqrt{E} x} & \text { if } x<1 \\ T_{N} \mathrm{e}^{\mathrm{i} \sqrt{E} x} & \text { if } x>N\end{cases}
$$

where $T_{N}$ and $R_{N}$ are the transmission and the reflection amplitudes of a system with $N$ scatterers, respectively. It is not difficult to compute recursively both amplitudes using well known transfer-matrix procedures (see, e.g., [21]). In particular, the transmission amplitude can be written as

$$
A_{N}=\left(\alpha_{N}+\frac{\alpha_{N-1}^{*} \beta_{N}}{\beta_{N-1}}\right) A_{N-1}-\left(\frac{\beta_{N}}{\beta_{N-1}}\right) A_{N-2}
$$

where $A_{N} \equiv 1 / T_{N}^{*}$, and

$$
\alpha_{j} \equiv\left[1-\mathrm{i}\left(\frac{1}{2 \sqrt{E}}\right) \lambda_{j}\right] \mathrm{e}^{\mathrm{i} \sqrt{E}} \quad \beta_{j} \equiv-\mathrm{i}\left(\frac{1}{2 \sqrt{E}}\right) \lambda_{j} \mathrm{e}^{-\mathrm{i} \sqrt{E}} .
$$

Finally, equation (8) must be supplemented by the initial condition $A_{0}=1, A_{1}=\alpha_{1}$ to completely determine the amplitudes.

Once we have computed the transmission amplitude, some physically relevant magnitudes can be readily obtained from it. Thus, the transmission coefficient is given by

$$
\tau_{N}=\left|T_{N}\right|^{2}
$$

whereas the resistivity, according to the Landauer formula [22], is simply

$$
\rho_{N}=\frac{1}{\left|T_{N}\right|^{2}}-1 \text {. }
$$

Aside from these two quantities, there are others that can also be obtained from the transmission amplitude, although somewhat less naturally. Indeed, the Lyapunov coefficient (which is nothing but the inverse of the localization length) depends on this amplitude through the expression [21]

$$
\gamma_{N}=-\frac{1}{2 N} \log \left|T_{N}\right|^{2}=-\frac{1}{2 N} \log \tau_{N}
$$

and it can also be shown [21] that the integrated density of states (IDOS) is related to $T_{N}$, by

$$
\Gamma_{N}=-\frac{\mathrm{i}}{2 \pi N} \log \frac{T_{N}^{*}}{T_{N}}
$$


From this last expression, the density of states (DOS) can be obtained by simple derivation with respect to the energy.

The results we have obtained so far provide an exact, although non-closed analytical description of any one-dimensional, disordered KP model. With them, we can compute the magnitudes we mentioned above for any given model and, in particular, for the CRDM. All expressions are very simple and suitable for an efficient numerical treatment of any specific case. We will now evaluate them for several of these cases to check whether there is any relevant feature of the transmission coefficient and related quantities that may be the fingerprint of extended states. We have to notice that there are several parameters that can be varied in the CRDM: the strengths of the two kinds of scatterers, $\lambda$ and $\lambda^{\prime}$, the defect concentration, and the length of the system, $N$. Without loss of generality, we fix $\lambda=1$ from now on and consider $\lambda^{\prime} \geqslant 1$ so that $(6 b)$ holds.

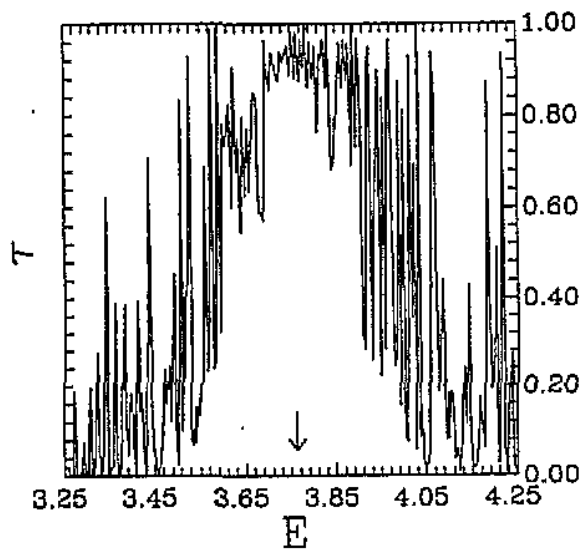

Figure 1. Transmission coefficient versus energy for a system with $\lambda^{\prime}=1.5,5000$ scatterers, and a probability of having a dimer of 0.5 . The arrow marks the predicted resonance.

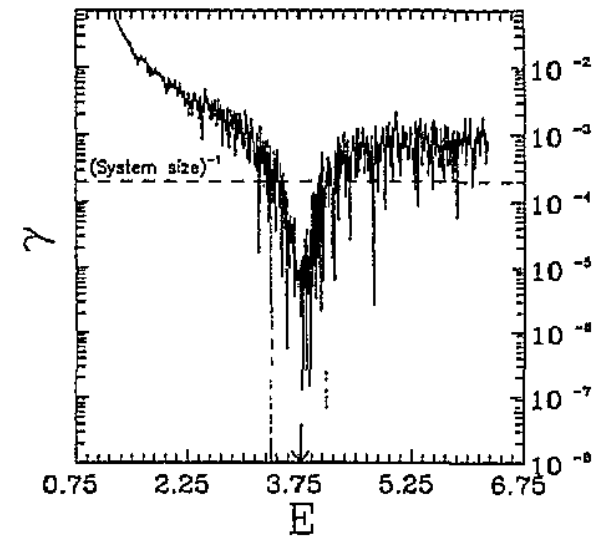

Figure 2. Lyapunov exponent versus energy for a system with $\lambda^{\prime}=1.5,5000$ scatterers, and a probability of having a dimer of 0.5. The dashed line marks the inverse of the system length: Energies with a lower exponent will have a localization length larger than the system length. The arrow marks the predicted resonance.

We first describe our results realizationwise, because we believe that these are the most physically relevant; we deal briefly with the average properties of the model below. In figure 1, we show the transmission coefficient for a system with $\lambda^{\prime}=1.5,5000$ scatterers, and a probability of having a dimer $q=0.5$. Each site is assigned a value $\lambda^{\prime}$ with probability $q$ and the next one is immediately set to $\lambda^{\prime}$ too; otherwise, with probability $1-q$ the site is given the value $\lambda$, and the next one is again chosen at random in the same way. The value $q$ does not coincide with the concentration of $\lambda^{\prime} s$, however, because if a site happens to be part of a dimer, the next one is already part of the dimer. To be specific, the ratio between the number of sites with strength $\lambda^{\prime}$ and $N$ is given by $2 q /(1+q)$. In this plot, it is clearly appreciated that the peak in the transmission coefficient is very close to the predicted value for the first resonance ( $E \approx 3.7626$ for this parameter set). Moreover, neighbouring states have a transmission coefficient close too unity, which decreases as we move away from the resonance. In figure 2, the Lyapunov exponent is plotted versus the energy for the same system; again, we appreciate that there is a deep minimum around the resonance value, which implies a very large localization length, much larger than the system size. The other 
magnitude we study, the resistivity; confirms the existence of an energy interval for which a typical realization of our model shows transport properties similar to those of perfect lattices.

The IDOS, which is plotted in figure 3, deserves some separate comments. Due to the presence of the multivalued log function in the defining relationship (13), this magnitude is very sensitive to the resolution in energies: if there is a jump in the arctan between two points for which the IDOS is computed, this jump will be missed and the IDOS will subsequently be underestimated. However, we checked several cases computing the IDOS with tiny energy steps $\left(5 \times 10^{-6}\right)$ which is very time consuming; with this accuracy, we recover the agreement between systems of different sizes (notice that the magnitude we discuss is in fact the IDOS per volume) as regards the total number of states and the IDOS structure. As to this last feature, we want to stress that the IDOS is well behaved (smooth) over all the studied range of energies. This implies that the same argument used by Dunlap et al $[10]$ to show that $\sqrt{N}$ states were extended holds in this case too, because the reasoning depends crucially on the DOS structure (see [15]).

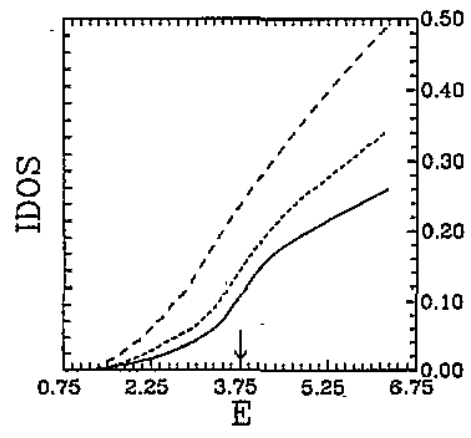

Figure 3. Integrated density of states for systems with $\lambda^{\prime}=1.5$ and a probability of having a dimer of 0.5 , of sizes 1000,5000 , and 10000 scatterers from top to bottom. The arrow marks the predicted resonance.

It is most important to report on how the above picture is modified when the system parameters are changed. First of all, the main characteristic of our model, i.e. that it has an infinite number of resonances, is confirmed by our calculations; besides, the higher the resonant energy (meaning the higher $n$ in $[(2 n-1) \pi / 2,(2 n+1) \pi / 2]$, the wider the peak in the transmission coefficient and the other transport magnitudes. The peak width also increases when decreasing $\lambda^{\prime}$ towards $\lambda=1$, and decreases when increasing $\lambda^{\prime}$. This is to be expected, because when $\lambda^{\prime}=\lambda$ we recover the perfect lattice. With respect to the other parameters, the number of scatterers and the concentration of dimers, both cause a narrowing of the set of extended states when they are increased in the range studied $(100 \leqslant N \leqslant 50000,0.1 \leqslant q \leqslant 0.5)$, although it is important to stress that this set always has non-zero width. Interestingly, when the number of scatterers increases, the IDOS steepens (i.e. the DOS exhibits a sensitive increment) around the resonant energy; consequently, the number of extended states may be constant in spite of the decreasing of the width of the transmission peak.

We now comment on the averaged results. When computing averages, they were taken over a number of realizations varying from 100 to 10000 to check the convergence of the computed mean values. The convergence was always satisfactory, with discrepancies of less than $1 \%$ between all the ensembles. Once more, however, to get accurate results for the IDOS is quite time consuming due to the necessary resolution in energies. The averaged results for the transmission coefficient, the Lyapunov exponent, the resistivity, and the density of states are basically the same as those for a typical realization commented on above. This is a crucial point because it supports our claim that those are the main features of our model irrespective of the particular realization of the disorder. 
In summary, we have studied a Kronig-Penney model with two kinds of sites, one of them constrained to appear only pairwise. We find an infinite number of energies for which the reflection coefficient of a single defect vanishes. We have shown, through numerical evaluation of exact expressions, that these resonances give rise to a very large number of extended states. These extended states are characterized by a transmission coefficient close to unity and a localization length much larger than the system length. The basis for the existence of extended states, relevant to affecting the transport properties and the smooth character of the DOS around the resonance [10,15], holds, supporting our conclusions. The increasing of the DOS around the resonance for large systems helps keep relevant the number of extended states. As a conclusion, we want to stress that the fact that we have more than one resonance is very important. Notice that the resonant energy values do not depend at all on the impurity concentration. Therefore, by modifying this concentration, we could shift the Fermi level of the system to match one of the resonances, either the one above or the one below its previous position. In this case, when the Fermi level reaches the set of resonant states, a large electrical conductance peak should appear. This effect could be relevant in the physical contexts mentioned at the beginning of this paper, most interestingly in the case of layered superconductors [5] or in disordered superlattices [4]. This possibility may open new perspectives in the design of electronic devices as well as in the design of materials with special properties. Further theoretical work towards a more comprehensive understanding of these questions is needed in order to pursue experimental evidence of this suppression of localization.

\section{Acknowledgments}

We are deeply indebted to Sergey A Gredeskul for very helpful discussions on our calculations, as well as to Rainer Scharf for drawing our attention on the random-dimer modet. AS was partially supported by DGIC y T (Spain) project PB92-0248.

\section{References}

[1] de Kronig L R and Penney W G 1931 Proc. R. Soc. A 130499

[2] See, e.g., Lieb E and Mattis D C 1966 Mathematical Physics in One Dimension (New York: Academic)

[3] Jaros M 1989 Physics and Application of Semiconductor Microstructures (Oxford: Clarendon)

[4] Tuncel E and Pavesi L 1992 Phil. Mag. B 65213

[5] Tanaka $Y$ and Tsukada M 1989 Phys. Rev. B 404482

[6] Gonil A R, Rojo A G and Martínez E N 1986 Am. J. Phys. 541018

[7] Clerk G J and McKellar B H J 1990 Phys. Rey, C 411198

[8] Anderson P W 1958 Phys. Rev. 1091492

[9] Ziman J M 1979 Models of Disorder (Cambridge: Cambridge University Press)

[10] Dunlap D H, Wu H-L and Phillips P 1990 Phys. Rev. Lett. 6588

[11] Wu H-L and Phillips P 1990 J. Chem. Phys. 937369

[12] Wu H-L and Phillips P 1991 Phys. Rev. Lett. 661366

[13] Phillips P and Wu H-L 1991 Science 2521805

[14] Flores J C 1989 J. Phys.: Condens. Matter 18471

[15] Bovier A 1992 J. Phys. A: Math. Gen. 251021

[16] Gangopadhyay S and Sen A K 1992 J. Phys. C: Solid State Phys. 49939

[17] Wu H-L, Goff W and Phillips P 1992 Phys. Rev. B 451623

[18] Erd6s P and Hendon R C 1977 Helv. Phys. Acta 50513

[19] Bellissard J, Formoso A, Lima R and Testard D 1982 Phys. Rev. B 263024

[20] Sokoloff J B and José J V 1982 Phys. Rev. Lett. 49334

[21] Kirkman P D and Pendry J B 1984 J. Phys. C: Solid State Phys. 174327

[22] Landaver R 1970 Phil. Mag. 21863 